\title{
Advanced analysis of high-temperature failure mechanisms in telecom lasers
}

\author{
Joachim Piprek \\ Electrical Engineering Department, University of California, Santa Barbara, CA 93106
}

\begin{abstract}
Laser performance degradation at elevated temperature often requires the use of costly cooling devices. Much effort has been devoted to understand and overcome the high-temperature failure of laser diodes used in telecommunication applications (wavelength 1.3-1.6 $\mu \mathrm{m}$ ). Various physical mechanisms have been proposed to explain high-temperature effects, including Auger recombination, carrier leakage, intervalence-band absorption, gain reduction and others. The discussion of the dominating effects is still controversial. One reason for this controversy is the use of simplified theoretical models that emphazise selected mechanisms. One-sided models lead to onesided interpretations of measurements. In this paper, high-temperature measurements on InP laser diodes are analyzed using a comprehensive laser model that includes all relevant physical mechanisms self-consistently. The software combines two-dimensional carrier transport, heat flux, strained quantum well gain computation, and optical wave guiding with a longitudinal mode solver. Careful adjustment of material parameters leads to an excellent agreement between simulation and measurements at all temperatures. At lower temperatures, Auger recombination controls the threshold current. At high temperatures, vertical electron leakage from the separate confinement layer is the main cause of performance degradation. The increase of internal absorption is less important. However, all these carrier and photon loss enhancements with higher temperature are mainly triggered by the reduction of the optical gain due to Fermi spreading of carriers.
\end{abstract}

Keywords: laser diode, long-wavelength semiconductor laser, InGaAsP/InP, temperature effects, failure mechanism, loss mechanism, Auger recombination, carrier leakage, intervalence-band absorption, laser gain, numerical simulation

\section{INTRODUCTION}

The strong temperature sensitivity of InP-based long-wavelength laser diodes has been investigated for more than two decades. ${ }^{1}$ Elevation of the external and/or internal laser temperature leads to reduced output power, higher threshold current, and lower slope efficiency. However, the dominating physical mechanisms responsible for such performance degradation are still under discussion. In recent years, this discussion includes Auger recombination, ${ }^{2}$ intervalence band absorption (IVBA), ${ }^{3}$ thermionic carrier emission out of the active region, ${ }^{4}$ lateral carrier spreading, ${ }^{5}$ passive layer absorption, ${ }^{6}$ spontaneous recombination within passive layers, ${ }^{7}$ and optical gain reductions. ${ }^{8,9}$ It was recently found that the non-uniformity of the MQW carrier distribution strongly affects the differential internal efficiency of long-wavelength multi-quantum well (MQW) lasers. ${ }^{10}$ All of the above mechanisms should be considered in the analysis of measurements. One-sided models lead to one-sided interpretations of experiments and contribute to the controversy in this field.

Advanced numerical laser models have been developed by several research groups. The more comprehensive these models are the more material parameters are involved. Accurate data for material parameters of ternary and quaternary semiconductor compounds are not always available. The composition of active region materials is often not exactly known. Some parameters may depend on the growth conditions. Thus, careful adjustment of material parameters used in the model is required to find agreement with measurements. Simultaneous reproduction of several experimental results is often necessary to analyze the relative importance of different mechanisms. The number of uncertain material parameters should be kept as small as possible by simplifying the experimental situation simulated. Pulsed laser operation at different stage temperatures, for instance, can be used to avoid self-heating of the device and to exclude heat flux from the model.

Email: piprek@ece.ucsb.edu, Telephone: 1-805-893-4051, Fax: 1-805-893-5440 


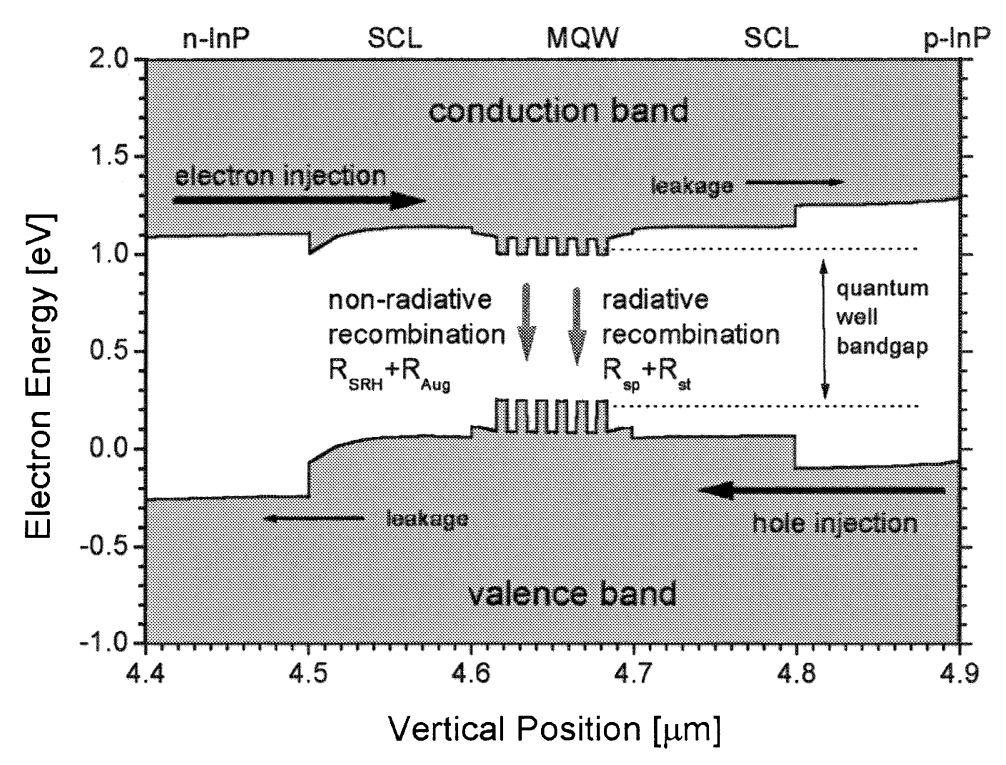

Figure 1. Energy band diagram of the InGaAsP/InP MQW laser diode investigated.

Sophisticated laser simulation tools are available today but a comprehensive agreement with measurements is hardly achieved. Using the commercial laser diode simulator PICS3D by Crosslight Software, this paper presents a numerical analysis of pulsed laser measurements that considers all of the above physical mechanisms and their interaction self-consistently. Good agreement with measurements is obtained by calibration of model parameters. Studying the performance degradation with increasing ambient temperature, high-temperature failure mechanisms are identified.

\section{DEVICE STRUCTURE}

A typical InGaAsP multi-quantum well (MQW) laser grown on InP is used as example device. ${ }^{11}$ The electron energy band diagram is given in Fig. 1. The MQW active region consists of six $6.4 \mathrm{~nm}$ thick compressively strained (1\% ) $\mathrm{In}_{0.76} \mathrm{Ga}_{0.24} \mathrm{As}_{0.79} \mathrm{P}_{0.21}$ quantum wells. The $5.5 \mathrm{~nm}$ thick barriers are made of $\operatorname{In}_{0.71} \mathrm{Ga}_{0.29} \mathrm{As}_{0.55} \mathrm{P}_{0.45}$ and exhibit slight tensile strain (0.04\%). The first and the last barrier are $17 \mathrm{~nm}$ wide. The MQW stack is sandwiched between undoped $100 \mathrm{~nm}$ thick InGaAsP separate confinement layers (SCLs) which act as waveguide layers. On the p-side of the structure, the first $140 \mathrm{~nm}$ of the $2000 \mathrm{~nm}$ InP cladding layer next to the SCL are not intentionally doped. The remainder of the InP layer is $4 \times 10^{17} \mathrm{~cm}^{-3} \mathrm{Zn}$ doped. Further details of the laser structure are given in Tab. 1. Broad area Fabry-Perot lasers with $W=57 \mu \mathrm{m}$ ridge width and $L=263 \mu \mathrm{m}$ cavity length are studied in this paper.

\section{MODELS AND PARAMETERS}

Fig. 1 illustrates the injection of electrons and holes into the MQW active layer as well as different recombination processes. Crystal defects and the Auger process cause non-radiative recombination. Photons are generated by spontaneous and by stimulated recombination. Carriers may also leak out of the waveguide region. Vertical leakage is typically dominated by electrons and lateral leakage by ambipolar carrier diffusion within the quantum wells (Fig. 2). Lateral carrier diffusion is illustrated in Fig. 2. Carriers leaving the waveguide region (gray area 
Table 1. Layer structure and parameters of the MQW broad-area laser investigated ( $l$ - layer thickness, $N_{d o p}$ - doping, $\mu$ - majority carrier mobility, $n$ - refractive index). ${ }^{12}$

\begin{tabular}{|l|l|l|l|l|}
\hline Parameter & $l$ & $N_{\text {dop }}$ & $\mu$ & $n$ \\
\hline Unit & $\mu \mathrm{m}$ & $1 / \mathrm{cm}^{3}$ & $\mathrm{~cm}^{2} / \mathrm{Vs}$ & \\
\hline \hline p-InP (cladding) & 1.86 & $4 \times 10^{17}$ & 100 & 3.17 \\
\hline $\mathrm{p}-\mathrm{InP}$ (cladding) & 0.14 & - & 150 & 3.17 \\
\hline $\mathrm{In}_{0.83} \mathrm{Ga}_{0.17} \mathrm{As}_{0.37} \mathrm{P}_{0.63}$ (SCL) & 0.1 & - & 100 & 3.28 \\
\hline $\mathrm{In}_{0.76} \mathrm{Ga}_{0.24} \mathrm{As}_{0.79} \mathrm{P}_{0.21}$ (QW) & 0.0064 & - & 100 & 3.42 \\
\hline $\mathrm{In}_{0.71} \mathrm{Ga}_{0.29} \mathrm{As}_{0.55} \mathrm{P}_{0.45}$ (barrier) & 0.0055 & - & 100 & 3.34 \\
\hline $\mathrm{In} 0.83 \mathrm{Ga}_{0.17} \mathrm{As}_{0.37} \mathrm{P}_{0.63}$ (SCL) & 0.1 & - & 100 & 3.28 \\
\hline $\mathrm{n}-\mathrm{InP}($ cladding) & 1.5 & $8 \times 10^{17}$ & 4500 & 3.17 \\
\hline n-InP (substrate) & 300 & $6 \times 10^{18}$ & 4500 & 3.17 \\
\hline
\end{tabular}

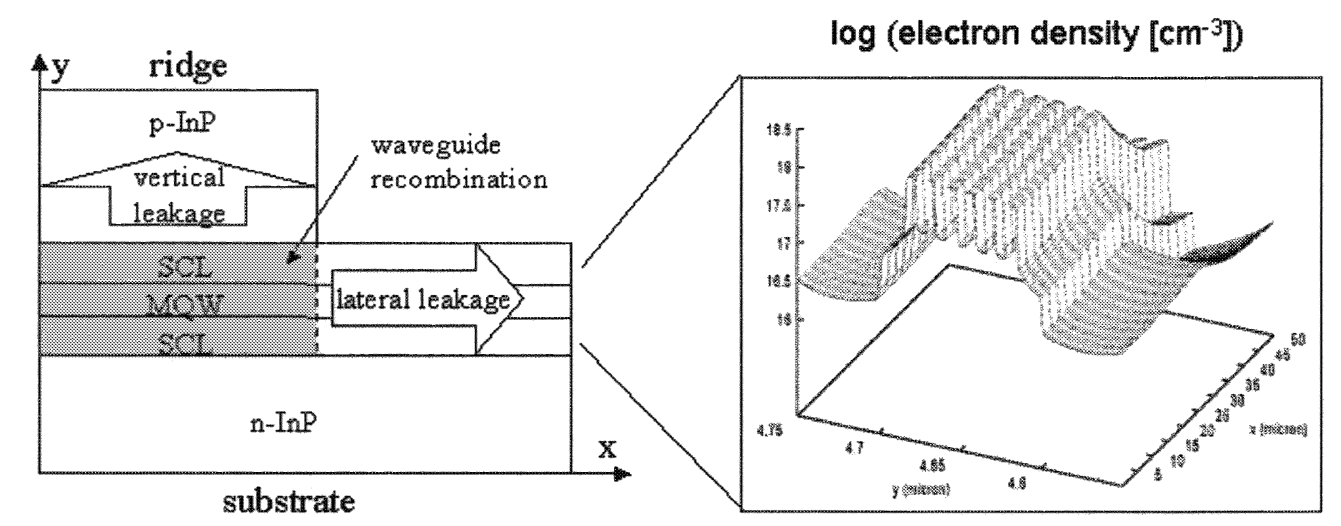

Figure 2. Left: half cross section of ridge-waveguide laser indicating leakage currents. Right: Electron Distribution in activer layers.

in Fig. 2) eventually recombine, however, they are considered leakage carriers in our analysis. Recombination mechanisms inside the waveguide region plus both the leakage currents add up to the total injection current. All carriers that do not contribute to stimulated recombination are considered lost carriers. The threshold current $I_{t h}$ needs to compensate for all the carrier losses at lasing threshold $\left(I_{r}\right.$-recombination losses, $I_{v}$-vertical leakage, $I_{l}$ - lateral leakage)

$$
I_{t h}=I_{r}+I_{v}+I_{l}
$$

Another important laser parameter is the slope efficiency above threshold $d P / d I[\mathrm{~W} / \mathrm{A}]$. It gives the differ- 
ential quantum efficiency

$$
\eta_{d}=\eta_{i} \frac{\alpha_{m}}{\alpha_{m}+\alpha_{i}}
$$

which depends on the internal optical losses $\left(\alpha_{i}\right)$ and on the enhancement of carrier losses above threshold $\left(\eta_{i}\right)$. The differential internal efficiency $\eta_{i}=\Delta I_{\text {stim }} / \Delta I$ is the fraction of the total current increment $\Delta I$ above threshold that results in stimulated emission of photons. ${ }^{13}$ It is less than unity if parts of $\Delta I$ are consumed by other recombination processes $\left(\Delta I_{r}\right)$ or by leakage $\left(\Delta I_{v}, \Delta I_{l}\right)$. The total current increment is the sum of all these contributions $\Delta I=\Delta I_{\text {stim }}+\Delta I_{r}+\Delta I_{v}+\Delta I_{l}$. The corresponding differential efficiencies are given as

$$
\begin{aligned}
\eta_{i} & =\eta_{l} \times \eta_{v} \times \eta_{r} \\
& =\frac{\Delta I_{\text {stim }}+\Delta I_{r}+\Delta I_{v}}{\Delta I_{\text {stim }}+\Delta I_{r}+\Delta I_{v}+\Delta I_{l}} \times \frac{\Delta I_{\text {stim }}+\Delta I_{r}}{\Delta I_{\text {stim }}+\Delta I_{r}+\Delta I_{v}} \times \frac{\Delta I_{\text {stim }}}{\Delta I_{\text {stim }}+\Delta I_{r}}
\end{aligned}
$$

These efficiencies can also be understood as probabilities. An additional electron injected above threshold has the probability $\eta_{l}$ to remain within the ridge region. It has the probability $\eta_{l} \eta_{v}$ to recombine within the waveguide region. The efficiency $\eta_{r}$ gives the ratio of the stimulated recombination increment to the total recombination increment within the waveguide region (incl. MQW). The difference between both the recombination rates is considered recombination loss. The recombination loss within SCLs and barriers is more than one order of magnitude smaller than within the quantum wells.

Laser diodes represent a complex interaction of electronic, thermal, optical, and quantum mechanical processes. The mathematical modeling of laser diodes needs to include all the physical mechanisms involved in a given problem. We will here focus on gain and loss mechanisms and their temperature dependence which can severely limit the performance of laser diodes. The model self-consistently combines carrier transport, optical gain computation, and wave guiding. It also involves many material parameters that are not exactly known. Critical parameters can be calibrated using measured laser characteristics as explained in the following paragraphs. Such calibration procedure is of paramount importance to draw realistic conlusions from the laser simulation. Pulsed LI characteristics measured at different temperatures are mainly used in this analysis. ${ }^{12}$

\subsection{Electrical Model}

The drift-diffusion model of carrier transport includes Fermi statistics and thermionic emission at hetero-barriers. This process is mainly controlled by the offset of conduction band $\left(\Delta E_{v}\right)$ and valence band $\left(\Delta E_{c}\right)$ at the hetero-barrier. Best agreement with high-temperature LI measurements is found by using a band offset ratio of $\Delta E_{c} / \Delta E_{v}=0.4 / 0.6$ which is typical for the InGaAsP/InP system. ${ }^{14}$ Lateral leakage is small in broad-area lasers and we employ the bulk mobilities given in Tab. 1. LI and VI measurements at different ridge widths can be used to identify lateral leakage ${ }^{15}$ and to calibrate critical parameters like the hole mobility. ${ }^{3}$ Within passive layers, a spontaneous emission parameter of $\mathrm{B}=10^{-10} \mathrm{~cm}^{3} \mathrm{~s}^{-1}$ is assumed. The spontaneous recombination rate in quantum wells is much larger than in passive layers and it is calculated self-consistently from energy band structure and Fermi distribution including temperature effects. Typical numbers are assumed for the ShockleyRead-Hall (SRH) recombination lifetime of electrons and holes with $20 \mathrm{~ns}$ inside the active region and 100 ns elsewhere. Due to the small QW bandgap, Auger recombination is known to cause the strongest carrier losses in long-wavelength lasers. Various theoretical and experimental investigations of this mechanism been published. ${ }^{8,16,17}$ By definition, the experimental Auger parameter $\mathrm{C}$ is somewhat different from the theoretical parameters $C_{n}$ and $C_{p}$ used in calculations of the local Auger recombination rate $\left(C_{n} n+C_{p} p\right)\left(n p-n_{i}^{2}\right)$. Seki et al. have investigated this difference for InP lasers similar to our example. ${ }^{17}$ Assuming that the valence-band CHHS Auger process dominates $\left(C_{n}=0\right)$, they reproduce experimental results employing an Arrhenius type function for the parameter $C_{p}=C_{o} \exp \left(-E_{a} / k T\right)$ with an activation energy of $E_{\mathrm{a}}=60 \mathrm{meV}$. We adopt this concept here using the same activation energy which gives good agreement with LI measurements at different temperatures. The fit leads to an Auger parameter of $C_{p}=1.6 \times 10^{-28} \mathrm{~cm}^{6} \mathrm{~s}^{-1}$ at room temperature which rises to $2.9 \times 10^{-28} \mathrm{~cm}^{6} \mathrm{~s}^{-1}$ at $120^{\circ} \mathrm{C}$. 

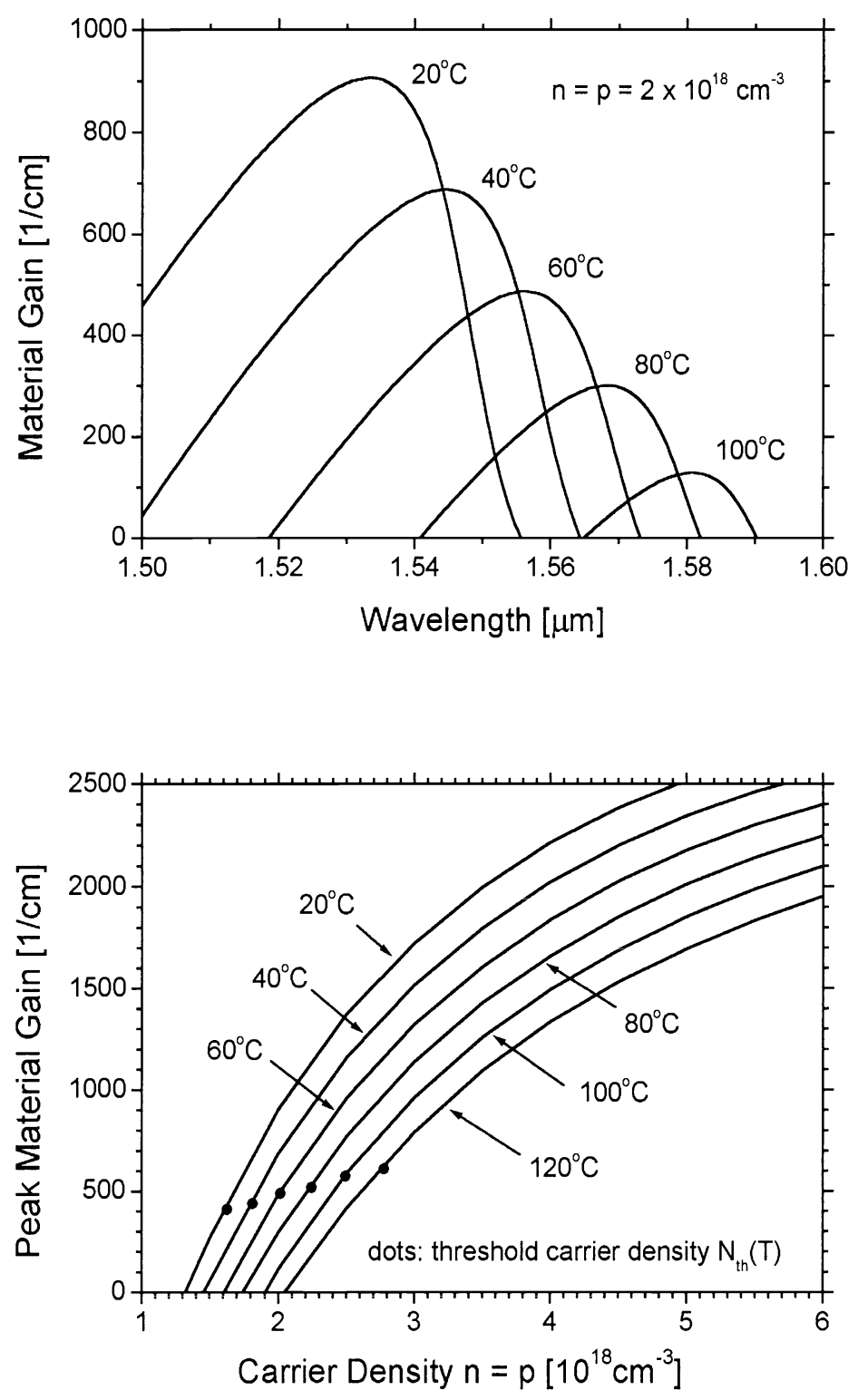

Figure 3. Top: net gain spectrum at constant carrier density $\left(n=p=2 \times 10^{18} \mathrm{~cm}^{-3}\right)$. Bottom: peak gain vs. carrier density. Parameter: ambient temperature. 


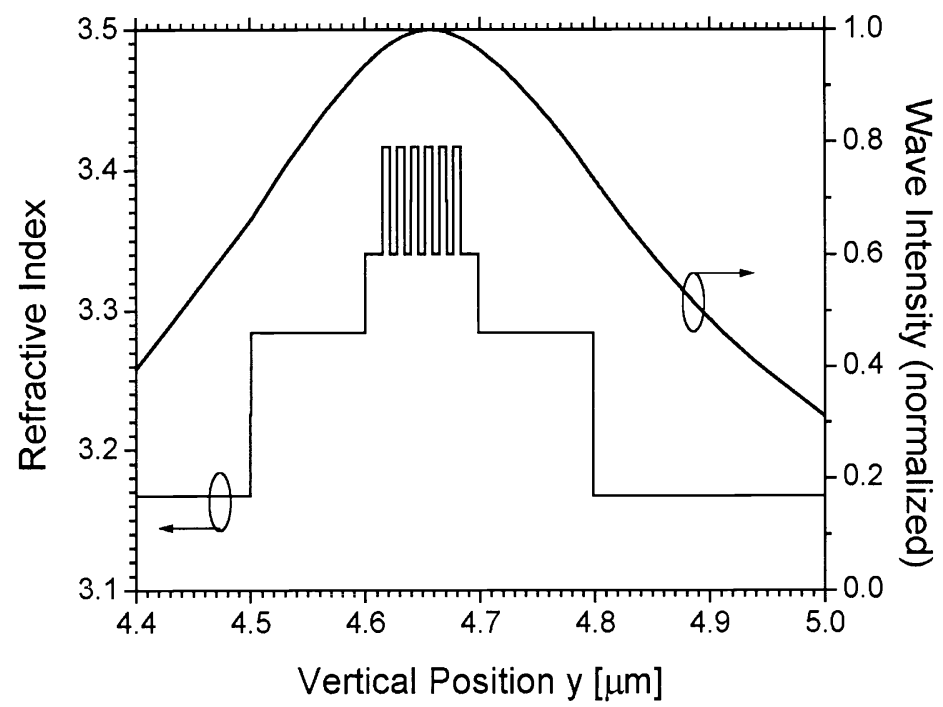

Figure 4. Vertical profile of refractive index and optical intensity.

\subsection{Gain Model}

For the strained QWs, the conduction bands are assumed to be parabolic and the non-parabolic valence bands are computed by the $4 \times 4 \mathrm{kp}$ method including valence band mixing. ${ }^{18}$ The local optical gain is calculated self-consistently from the local Fermi distribution of carriers at each bias point of the LI curve. A Lorentzian broadening function is used with 0.1 ps intraband relaxation time. Band gap shrinkage due to carrier-carrier interaction is considered as $\Delta E_{g}=-\xi N^{1 / 3}$ with $\xi=10^{-8} \mathrm{eV} \mathrm{cm}$. The thermal band gap reduction parameter $d E_{g} / d T=-0.28 \mathrm{meV} / \mathrm{K}$ is extracted from the measured thermal shift of the lasing wavelength. This number is in agreement with measurements of the photoluminescence peak shift of similar MQWs. ${ }^{19}$ Temperature effects on the calculated gain spectrum are shown in Fig. 3 with constant carrier density. With higher temperature, the peak gain decreases substantially due to the wider spreading of the Fermi distribution of carriers. Figure 3 also plots the peak gain as function of the carrier density at different temperatures. To maintain the required threshold gain with rising temperature, carrier density and injection current need to be increased. As shown below, this is the main trigger mechanism for the strong temperature sensitivity of the threshold current.

\subsection{Optical Model}

The refractive index profile of our structure is listed in Tab. 1 and plotted in Fig. 4 together with the optical intensity. The optical confinement factor is $\Gamma=0.074$. The local absorption coefficient is proportional to the density of electrons $(n)$ and holes $(p): \alpha=\alpha_{b}+k_{n} n+k_{p} p$. The constant background loss coefficient $\alpha_{b}$ represents carrier-density independent mechanisms like photon scattering at defects. Free-carrier absorption due to electrons is known to be very small in $1.55 \mu \mathrm{m} \operatorname{InGaAsP} / \operatorname{InP}$ lasers $\left(k_{n} \approx 10^{-18} \mathrm{~cm}^{2}\right) .{ }^{1}$ Absorption within the valence bands can be related to intraband transitions (free-carrier absorption) or to intervalenceband transitions (IVBA). Both mechanisms are roughly proportional to the hole density and hard to separate in our analysis. IVBA is usually considered the dominant absorption mechanism in $1.55 \mu \mathrm{m}$ lasers. ${ }^{1}$ Absorption measurements at $1.55 \mu \mathrm{m}$ wavelength give $k_{p}=20 \ldots 60 \times 10^{-18} \mathrm{~cm}^{2}$ for bulk material (InGaAsP exhibits higher values than InP) ${ }^{20-22}$ It is difficult to accurately measure this parameter within quantum wells and only few experimental investigations of $k_{p}$ can be found in the literature on $1.55 \mu \mathrm{m}$ MQW structures. ${ }^{22,} 23$ IVBA is often believed to be reduced by compressive strain, but some investigations suggest otherwise. ${ }^{24,25}$ Quantum-well IVBA is hard to distinguish from IVBA in other layers and we assume $k_{p}$ and $k_{n}$ to be uniform throughout our device, i.e., carrier density dependent absorption within barriers and SCLs is included self-consistently. Our fit to LI measurements at different cavity length gives $k_{p}=82 \times 10^{-18} \mathrm{~cm}^{2}$ at room temperature. This number mainly represents the 


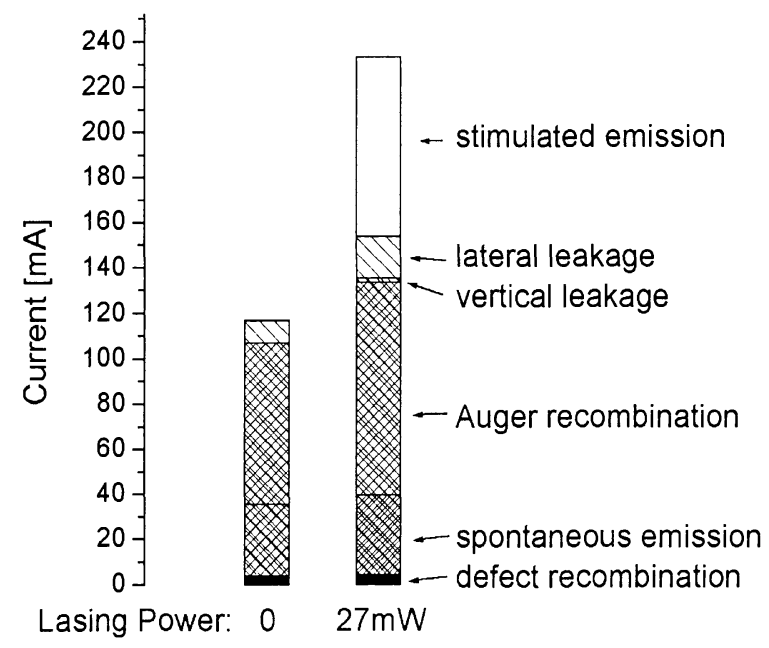

Figure 5. Contribution of carrier losses to the total current at threshold and at $27 \mathrm{~mW}$ output power.

quantum wells. It is smaller than measured with unstrained quantum wells $\left(140 \times 10^{-18} \mathrm{~cm}^{2}\right)$ and larger than with more strongly strained quantum wells $\left(35 \times 10^{-18} \mathrm{~cm}^{2}\right.$ with $1.2 \%$ strain $) .{ }^{22} \quad$ From LI characteristics at different temperature, we find a very weak temperature sensitivity of this parameter $\left(k_{p}=88 \times 10^{-18} \mathrm{~cm}^{2}\right.$ at $120^{\circ} \mathrm{C}$ ) which is in agreement with other investigations. ${ }^{9,20,21}$ The modal internal loss parameter $\alpha_{i}$ is obtained by $3 \mathrm{D}$ integration, weighted by the local intensity of the fundamental mode. Alternatively, $\alpha_{i}$ could also be used as fit parameter without detailed absorption assesment for each layer. However, this simple approach would not reflect temperature effects on $\alpha_{i}(\mathrm{~T})$ which are related to the local carrier density. For our lasers, vanishing background loss $\left(\alpha_{b}=0\right)$ and $\mathrm{R}=0.28$ facet power reflectance give best agreement with LI measurements at different laser length. ${ }^{26}$ The mirror loss coefficient is $\alpha=47 \mathrm{~cm}^{-1}$ for our cavity length of $\mathrm{L}=269 \mu \mathrm{m}$.

\section{ANALYSIS AT ROOM TEMPERATURE}

The differential quantum efficiency $\eta_{d}$ is one of the most important performance parameters of laser diodes. It gives the percentage of electrons injected above threshold that contributes photons to the emitted laser beam. This efficiency is restricted by carrier losses $\left(\eta_{i}\right)$ and by photon losses $\left(\alpha_{i}\right)$. The injection efficiency (or differential internal efficiency) $\eta_{i}$ is equal to the fraction of current above threshold that results in stimulated emission of photons. Low injection efficiency $\eta_{i}$ is usually attributed to vertical electron leakage which is known to escalate at higher temperatures. Additional electron stopper layers introducing a barrier in the conduction band have been used successfully to enhance the high-temperature performance of long-wavelength lasers. ${ }^{27}$ For our MQW laser, $\eta_{i}=67 \%$ was measured at room temperature which suggests strong electron leakage. ${ }^{11}$ Thus, a second laser structure was fabricated with an additional InGaP electron stopper layer between SCL and p-InP. ${ }^{11}$ The stopper layer has a conduction band offset to $\mathrm{InP}$ of $\Delta E_{c} \approx 50 \mathrm{meV}$ but it does not hinder hole transport. However, no significant change in the laser characteristics is measured at room temperature indicating negligible vertical leakage. ${ }^{11}$ Since both $\eta_{v}$ and $\eta_{l}$ seem to be close to $100 \%$ in these broad-area lasers, the experimental results direct the attention towards recombination losses $\left(\eta_{r}\right)$ to account for the reduced internal efficiency measured. Commonly, the QW carrier density is considered constant above threshold (Fermi level pinning), leading to $\eta_{r} \approx 100 \%$.

This phenomenon is analyzed by evaluating carrier loss mechanisms in our laser simulation at room temperature. Electron leakage from the active region can be identified as minority carrier current in the p-InP cladding layer. At threshold, far less than $1 \%$ of the total electron current leaks into the p-cladding, i.e., adding an 


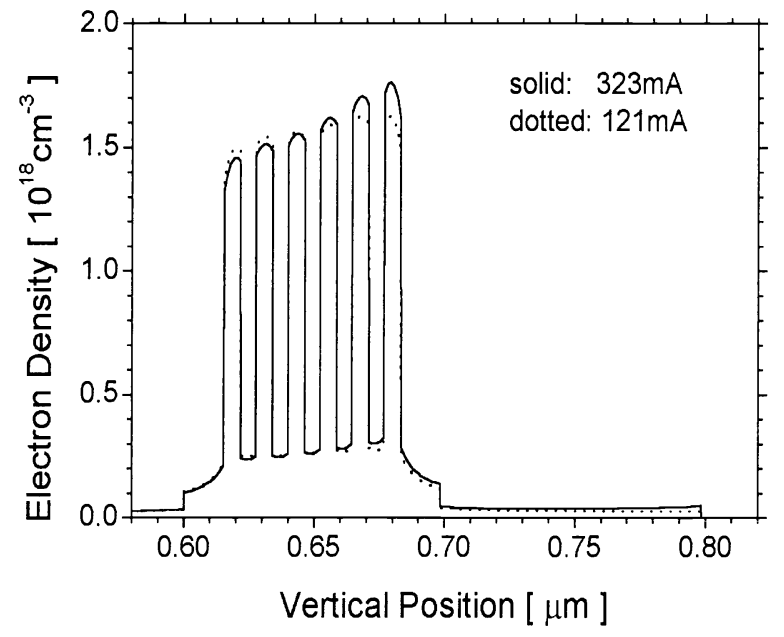

Figure 6. Vertical profile of the MQW electron density at different currents.

InGaP barrier cannot have any significant effect at room temperature. Hole leakage is even smaller. Lateral leakage accounts for about $8 \%$ of the threshold current. Carrier losses due to Auger recombination, spontaneous emission, and defect recombination are analyzed by integrating the recombination rates at different bias points. At threshold, $61 \%$ of the total current feeds Auger recombination, $27 \%$ spontaneous emission, and 3\% defect recombination. Figure 5 shows that all those carrier losses grow above threshold. This growth reduces the differential internal efficiency $\eta_{i}$, which would be $100 \%$ without it. The growth can largely be attributed to the increasing non-uniformity of the MQW carrier distribution (Fig. 6). The separation of quasi-Fermi levels in neighbor quantum wells increases with higher current due to the electrical resistance of the barrier. This causes an increment of Auger recombination within the more populated p-side quantum wells that is larger than the decrement in the less populated n-side quantum wells. Auger recombination and spontaneous emission exhibit a super-linear dependence on the local carrier density and increasing carrier non-uniformity causes stronger total recombination losses. SRH recombination grows linear with the local carrier density and it reflects only the change in average carrier density. The net increase of recombination losses results in $\eta_{r}=74 \%$ for our device. Using Eq. 4, we extract the leackage contributions $\eta_{v}=99 \%$ and $\eta_{l}=93 \%$. The total injection efficiency $\eta_{i}=68 \%$ is very close to the measured number and it is clearly dominated by recombination losses.

\section{HIGH-TEMPERATURE EFFECTS}

Threshold current and slope efficiency have been measured in pulsed operation within the ambient temperature range from $20^{\circ} \mathrm{C}$ to $120^{\circ} \mathrm{C} .{ }^{12}$ As result of the parameter calibration described above, the simulation shows excellent agreement with these measurements. Figure 7 plots the calculated threshold current and its components as function of temperature. All recombination currents are obtained by 3D integration over the waveguide region. Carriers leaving the waveguide region in lateral or vertical direction constitute leakage currents. At lower temperatures, the strongest contribution to the threshold current comes from QW Auger recombination, followed by spontaneous emission, lateral leakage current, and SRH recombination. Vertical carrier leakage is negligible at room temperature but it increases strongly and becomes the dominant carrier loss mechanism above $120^{\circ} \mathrm{C}$. Thus, the temperature sensitivity of the threshold current is dominated by Auger recombination at lower temperatures and by vertical leakage at higher temperatures.

The escalation of carrier losses with higher temperature is related to the increasing carrier density which is mainly triggered by the gain reduction with higher temperature (Fig. 3). This becomes clear from Fig. 


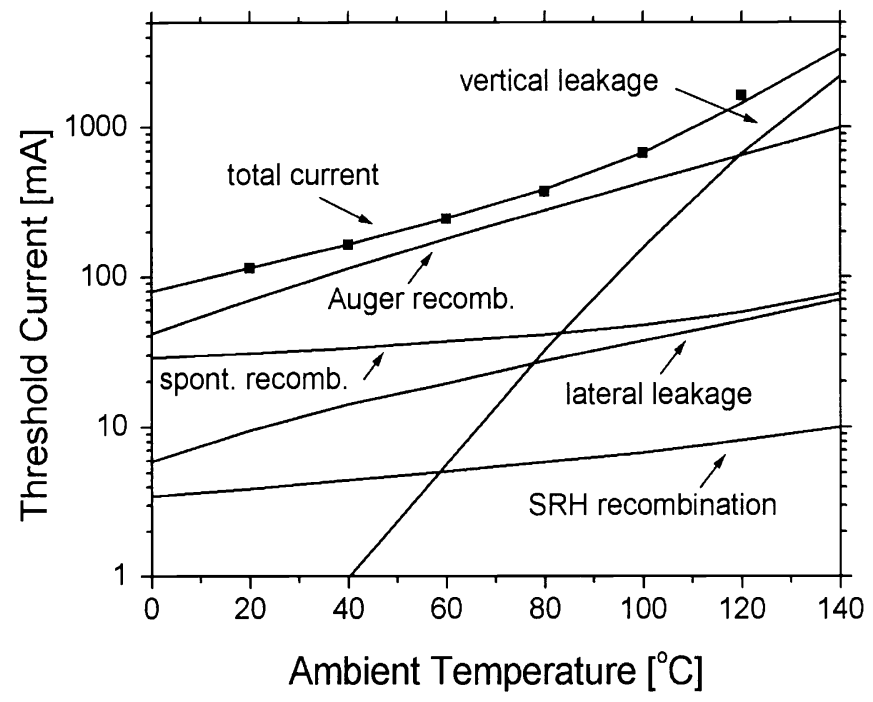

Figure 7. Pulsed threshold current and its components as function of ambient temperature (dots: measurement).

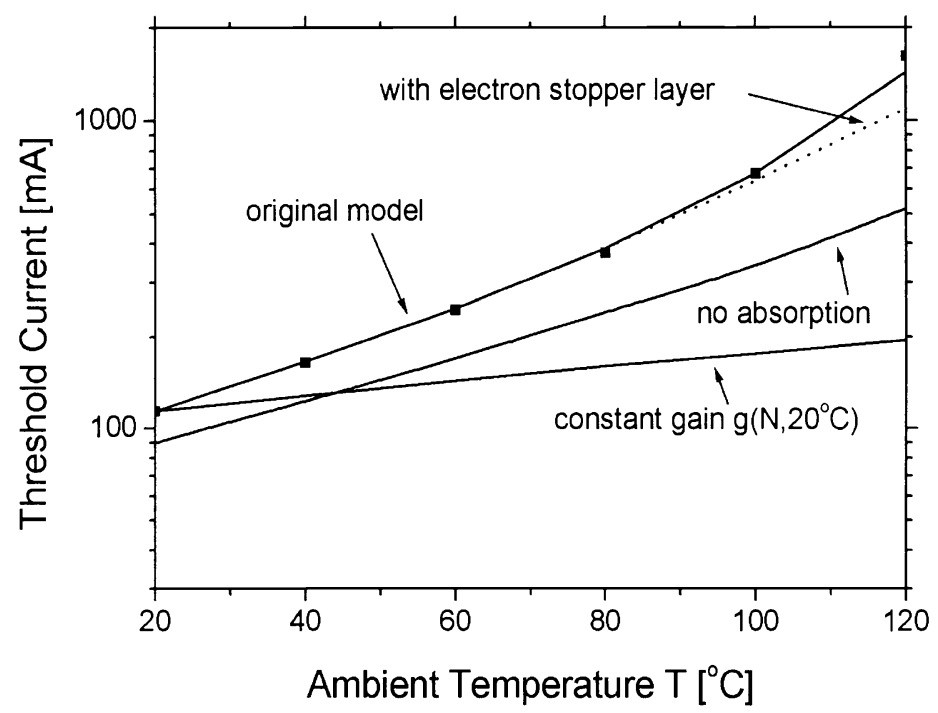

Figure 8. Threshold current as function of ambient temperature with model variations. 


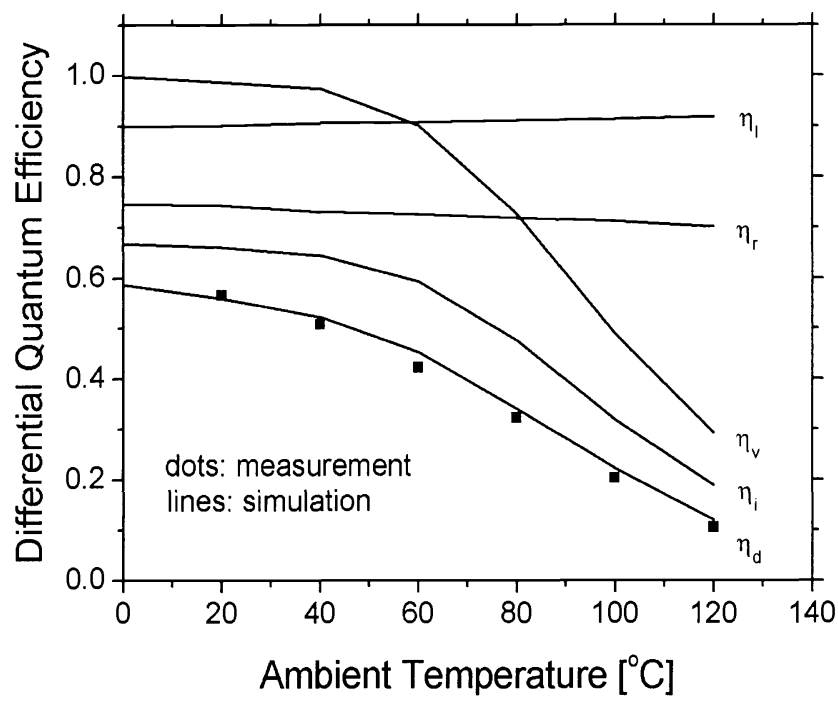

Figure 9. Slope efficiency and its components as function of ambient temperature (dots: measurements).

8. Assuming constant gain, the temperature sensitivity of the threshold current would be very small, despite Auger recombination. Thus, the reduced gain is the trigger mechanism for increasing carrier losses. This result confirms experimental investigations in $^{8,9}$ which have been disputed elsewhere. ${ }^{2}$ Figure 8 also shows the effects of other model variations. Including an electron stopper layer ${ }^{11}$ only affects the threshold current at high temperatures, when vertical leakage becomes relevant. Without absorption, the threshold current and it's temperature sensitivity are slightly reduced in Fig. 8. Obviously, absorption is not the dominating mechanism causing high-temperature failure, as suggested by other authors. ${ }^{28}$

Figure 9 plots the temperature sensitivity of the slope efficiency and of its components. Above $80^{\circ} \mathrm{C}$, electron leakage causes the highest differential carrier loss. The recombination loss increment $\Delta I_{r}$ rises little with higher temperature. This is related to the more uniform hole distribution which compensates for rising Auger recombination. Due to a lower carrier mobility, the lateral leakage increment $\Delta I_{l}$ decreases slightly thereby reducing the temperature sensitivity of the slope efficiency, as previously observed. ${ }^{15}$

\section{SUMMARY}

In our multi-quantum well laser, threshold current and slope efficiency are mainly governed by Auger recombination at low temperatures and by vertical electron leakage at high temperatures. The enhancement of carrier losses and internal absorption with rising temperature is mainly controlled by the increasing carrier density in active and passive layers. This increase is caused by optical gain reductions with higher temperature. Thus, only the self-consistent consideration of temperature effects on gain, carrier density, recombination, leakage, and absorption leads to a full explanation of the measured performance degradation at high temperatures.

\section{REFERENCES}

1. Y. Suematsu and A. R. Adams, eds., Handbook of Semiconductor Lasers and Photonic Integrated Circuits, Chapman \& Hall, London, 1994.

2. J. Braithwaite, M. Silver, V. A. Wilkinson, E. P. O'Reilly, and A. R. Adams, "Role of radiative and nonradiative processes on the temperature sensitivity of strained and unstrained $1.5 \mu \mathrm{m} \operatorname{InGaAs}(\mathrm{P})$ quantum well lasers," Applied Physics Letters 67, pp. 3546-3548, 1995.

3. J. Piprek, D. I. Babic, and J. E. Bowers, "Simulation and analysis of 1.55-micron double-fused vertical-cavity lasers," Journal of Applied Physics 81, pp. 3382-3390, 1997. 
4. L. J. P. Ketelsen and R. F. Kazarinov, "Carrier loss in InGaAsP-InP lasers grown by hydride CVD," IEEE Journal of Quantum Electronics 34, pp. 811-813, 1995.

5. Y. Yoshida, H. Watanabe, K. Shibata, A. Takemoto, and H. Higuchi, "Analysis of characteristic temperature for InGaAsP BH lasers with p-n-p-n blocking layers using two-dimensional device simulator," IEEE Journal of Quantum Electronics 34, pp. 1257-1262, 1998.

6. V. Mikhaelashvili, N. Tessler, R. Nagar, G. Eisenstein, A. G. Dentai, S. Chandrasakhar, and C. H. Joyner, "Temperature dependent loss and overflow effects in quantum well lasers," IEEE Photonics Technology Letters 6, pp. 1293-1296, 1994.

7. A. A. Bernussi, H. Temkin, D. Coblentz, and R. A. Logan, "Effect of barrier recombination on the high temperature performance of quaternary multiquantum well lasers," Applied Physics Letters 66, pp. 67-69, 1995.

8. Y. Zou, J. Osinski, P. Grodzinski, P. Dapkus, W. Rideout, W. Sharfin, J. Schlafer, and F. Crawford, "Experimental study of Auger recombination, gain, and temperature sensitivity of $1.5 \mu \mathrm{m}$ compressively strained semiconductor lasers.," IEEE Journal of Quantum Electronics 29, pp. 1565-1575, 1993.

9. D. A. Ackerman, G. E. Shtengel, M. S. Hybertsen, P. A. Morgan, R. F. Kazarinov, T. Tanbun-Ek, and R. A. Logan, "Analysis of gain in determining $\mathrm{T}_{\mathrm{o}}$ in $1.3 \mu \mathrm{m}$ semiconductor lasers," IEEE Journal of Selected Topics in Quantum Electronics 1, pp. 250-262, 1995.

10. J. Piprek, P. Abraham, and J. E. Bowers, "Carrier nonuniformity effects on the internal efficiency of multiquantum-well lasers," Applied Physics Letters 74(4), pp. 489-91, 1999.

11. P. Abraham, J. Piprek, S. DenBaars, and J. E. Bowers, "Study of temperature effects on loss mechanisms in $1.55 \mu \mathrm{m}$ laser diodes with InGaP electron stopper layer," Semiconductor Science and Technology 14, pp. 419-424, 1999.

12. J. Piprek, P. Abraham, and J. E. Bowers, "Self-consistent analysis of high-temperature effects on strainedlayer multi-quantum well InGaAsP/InP lasers," IEEE Journal of Quantum Electronics 36, pp. 366-374, 2000.

13. L. A. Coldren and S. W. Corzine, Diode Lasers and Photonic Integrated Physics, Wiley, New York, 1995.

14. S. L. Chuang, Physics of Optoelectronic Devices, Wiley, New York, 1995.

15. G. J. Letal, J. G. Simmons, J. D. Evans, and G. P. Li, "Determination of active-region leakage currents in ridge-waveguide strained-layer quantum-well lasers by varying the ridge width," IEEE Journal of Quantum Electronics 34, pp. 512-518, 1998.

16. G. Fuchs, C. Schiedel, A. Hangleiter, V. Harle, and F. Scholz, "Auger recombination in strained and unstrained InGaAs/InGaAsP multiple quantum well lasers," Applied Physics Letters 62, pp. 396- 398, 1993.

17. S. Seki, W.W.Lui, and K. Yokoyama, "Explanation for the temperature insensitivity of the Auger recombination rates in $1.55 \mu \mathrm{m}$ InP-based strained-layer quantum-well lasers," Applied Physics Letters 66, pp. 3093-3095, 1995.

18. S. Chuang, "Efficient band-structure calculation of strained quantum-wells," Physical Review B 43, pp. 9649- 9661, 1991.

19. S. Rapp, J. Piprek, K. Streubel, J. Andre, and J. Wallin, "Temperature sensitivity of 1.54 micron verticalcavity lasers with an InP-based Bragg reflector," IEEE Journal of Quantum Electronics 33(10), pp. 1839-45, 1997.

20. H. C. Casey and P. L. Panish, "Variation of intervalence band absorption with hole concentration in p-type InP," Applied Physics Letters 44, pp. 82-84, 1984.

21. C. H. Henry, R. A. Logan, F.R.Merritt, and J. P. Luongo, "The effect of intervalence band absorption on the thermal behavior of ingaasp lasers," IEEE Journal of Quantum Electronics 19, pp. 947-952, 1993.

22. I. Joindot and J.L.Beylat, "Intervalence band absorption coefficient measurements in bulk layer, strained and unstrained multiquantum well $1.55 \mu \mathrm{m}$ semiconductor lasers," Electronics Letters 29, pp. 604-606, 1993.

23. G. Fuchs, J. Hoerer, A. Hangleiter, V. Haerle, F. Scholz, R.W.Glew, and L. Goldstein, "Intervalence band absorption in strained and unstrained ingaas multiple quantum well structures," Applied Physics Letters 60, pp. 231-233, 1992. 
24. W.S.Ring, "Examinationof intervalence band absorption and its reduction by strain in $1.55 \mu \mathrm{m}$ compressively strained InGaAs/InP laser diodes," Electronics Letters 30, pp. 306-308, 1994.

25. T. Cho, H. Kim, Y. Kwon, and S. Hong, "Theoretical study on intervalence band absorption in InP-based quantum-well laser structures," Applied Physics Letters 68, pp. 2183-2185, 1996.

26. J. Piprek, P. Abraham, and J. E. Bowers, "Cavity length effects on internal loss and quantum efficiency of multi-quantum-well lasers," IEEE Journal of Selected Topics in Quantum Electronics 5, pp. 643-647, 1999.

27. R. F. Kazarinov and M. R. Pinto, "Carrier transport in laser heterostructures," IEEE Journal of Quantum Electronics 30, pp. 49-53, 1994.

28. S. Seki, H. Oohashi, H. Sugiura, T. Hirono, and K. Yokoyama, "Study on the dominant mechanism for the temperature sensitivity of threshold current in $1.3 \mu \mathrm{m} \mathrm{InP-based} \mathrm{strained-layer} \mathrm{quantum-well} \mathrm{lasers,"} \mathrm{IEEE}$ Journal of Quantum Electronics 32, pp. 1478-1486, 1996. 